\title{
Nigerian Vehicle License Plate Recognition System using Artificial Neural Network
}

\author{
Amusan D.G ${ }^{1}$, Arulogun O.T ${ }^{2}$ and Falohun A.S \\ Open and Distance Learning Centre, Ladoke Akintola University of Technology, Ogbomoso, Oyo State, Nigeria ${ }^{1}$ \\ Department of Computer Science and Engineering, Ladoke Akintola University of Technology, Ogbomoso, \\ Oyo State, Nigeria ${ }^{2,3}$
}

\begin{abstract}
In this paper, a development of Nigeria Vehicle License Plate Recognition (NVLPR) system using artificial neural network is done. Vehicle License Plate Recognition (VLPR) is a special form of optical character recognition (OCR) which enables computer systems to read automatically the registration number of vehicles from digital pictures for the purpose of traffic control, security, access control to restricted areas, tracking of cars, tracing of stolen cars, identification of dangerous and reckless drivers on the road. This system is divided into three major parts: vehicle license plate detection, vehicle license plate character segmentation and License Plate character recognition. In vehicle license plate detection there are many challenges such as, complex plate background, illumination in consistencies, vehicle motion, distance changes for which edge detection analysis was explored. In this work, 200 vehicle license plates were captured, some with clear characters, others with blur and dirty stains. The character feature extraction and plate recognition accuracies were determined. Results showed that plates without blur and stain were most accurately extracted and recognized while satisfactory results were also obtained for the others.
\end{abstract}

Keywords: Software, License plate detection and recognition, Optical Character Recognition (OCR), Nigerian vehicle license plate, artificial neural network.

\section{INTRODUCTION}

Vehicle license plate recognition system involves capturing of photographic video or images of license plates, whereby they are processed by a series of algorithms that are able to provide an alpha numeric conversion of the captured license plate images into a text entry. Vehicle license plate recognition is designed to identify and recognize the number plate of a moving vehicle automatically. Automatic Vehicle License Plate Recognition has a wide range of applications such as access control whereby a gate automatically opens for authorized members in a secured area, tracking of car and tracing of stolen cars, law enforcement the manual process of preparing a violation fine is replaced by an automated process which reduces the overhead and turnaround time and border control. Vehicle License Plate Recognition (VLPR) is a form of automatic vehicle identification. It is an image processing technology used to identify vehicles only by their license plates. Real time LPR plays a major role in automatic monitoring of traffic rules and maintaining law enforcement on public roads [1].

VLPR has been a good area of research interest due to its vast area of application. It has been divided into three major parts; the first part involves vehicle license plate detection: edge detection technique and extraction method; Second part is character segmentation which is achieved by vertical and horizontal projection analysis. Third step is Character Recognition: the numbers and the letters are classified using ANN. The character recognition part of the system is carry out by using artificial neural network [2].This task is quite challenging due to the diversity of plate formats, the non-uniform outdoor illumination conditions, complex background, and distance change during image acquisition [3]. To overcome these problems image enhancement method is applied and also median filter is used on the images to remove noise and unwanted effect of light in order to obtain clear and readable images [4]. This paper simulated a plate recognition system which is capable of identifying and recognizing Nigerian vehicle license plates.

In Nigeria the attributes of the vehicle license plates are strictly maintained. For example, the size of the plate, colour of the plate, font face/ size/ colour of each character, spacing between subsequent characters, the number of lines in the plates, script etc. [4]. The styles of vehicle license plate that are considered in this paper are shown in Figure 1. It shows various styles of license plates found on vehicles in Nigeria. Each style is associated with a particular class of vehicle. The classes include private vehicles and commercial vehicles. Other categories of vehicles, such as official cars and military cars are not addressed since they are rarely seen.

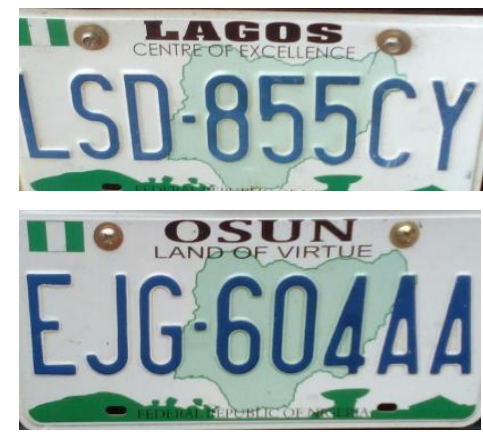




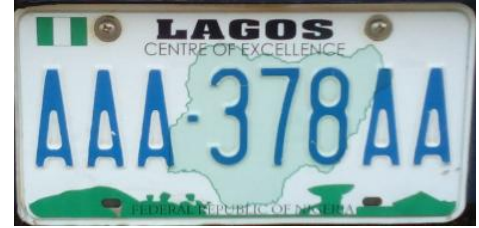

(a)

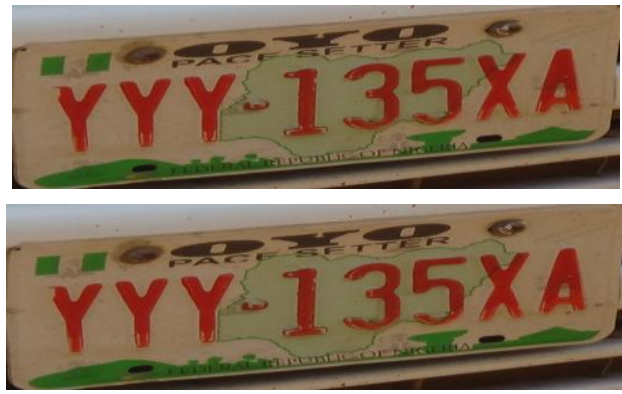

(b)

Figure 1: Standard vehicle plate used in Nigeria: (a) private (b) commercial.

A brief description of the proposed (NVLPR) system is as shown in Figure 2.

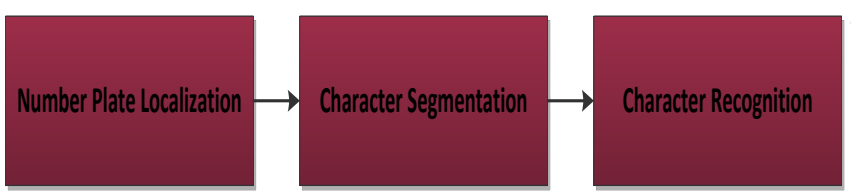

Figure 2: License plate recognition system.

\section{RELATEDWORK}

Many research works have been carry out in the field of vehicle license plate recognition. Diegert C [5] suggested an approach to trace the trucks and automobile from overhead images view, called a shape first approach. This approach tested on Overhead Imaging Research Dataset (OIRDS). The tracing process utilized the information from overhead image of an automobile, and recognizing the candidate vehicles from outlines of their shapes. On the other hand, Sarfraz, M. et al. [6] utilized vertical edge detection and filtering which is then followed by vertical edge matching in the localization of Saudi Arabian license plates. As it is observed that images have more horizontal lines than vertical lines, this approach reduces computation time by detecting only vertical lines.

In addition, [7] used image texture feature to locate license plate from the entire image and [8] presented a vehicle segmentation method to detect, track and classify moving vehicles in presence of occlusion in crowded scenario. The authors used a vehicle outline patterns, camera adjustment and ground plane information for detection process instead of blob tracking methods due to its inability to separate vehicles under occlusion. Different techniques are used by different researchers in detection of license plates, such as the methods based on colour feature [7], edge extraction [8], histogram analysis [9], and morphological operators [10].In recognizing Chinese license plates a method called structural verification was used for plate location and projection segmentation is exploited for incising plate image into character images. Sobel operator is the first and best known choice for most license plate detection algorithms to convert the grayscale scene image to the gradient image at the initial stage.

\section{RESEARCHMETHODOLOGY}

The developed recognition system aims to identify and recognize Nigeria vehicle license plates. The input to the system is the image of the vehicle acquired by a digital camera and the output of the system is the detected and recognized license plate. The implementation of the program is developed on MATLAB 8.0.

The implementation processe s are as follows:

\subsection{Pre-processing}

This is a process where the colour input image is being converted to gray scale image, the character is binarized and the noise is eliminated in the preprocessing stage.

- Gray scale conversion

Vehicle image has different colours the system then converts the RGB images to grayscale images using NTSC standard method. Gray $=0.299 *$ Red $+0.587 *$ Green $+0.114 *$ Blue.

- Gaussian Filtering

It is very certain that during image acquisition there are different types of noise that comes with original image. Gaussian filtering was used to eliminate the noises. Using Gaussian filtering not only can eliminate the noises, but also make the high frequency more concentrated. There by, it is beneficial for us to detect the edges in images. The salt and pepper characteristic of vehicle license plate is presented, So Gaussian filter was adopted for removing such noise.

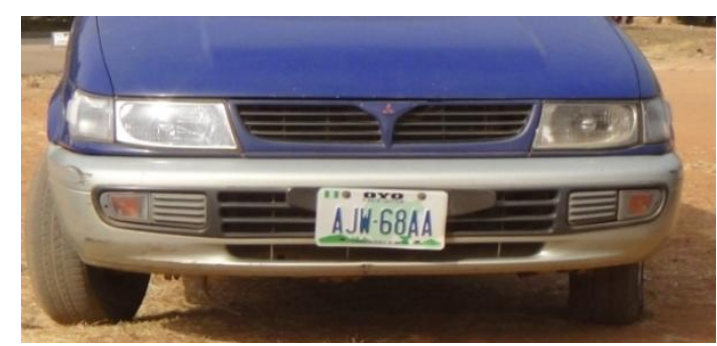

Figure 3(a): input image

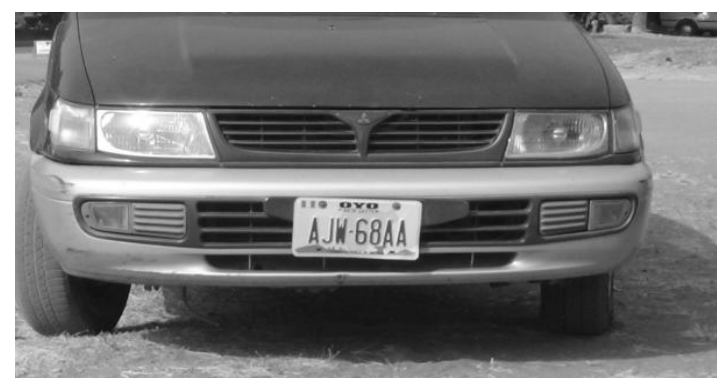

Figure 3(b): Gray scale image

\subsection{Plate Localization}

To obtain a reliable localization of license plate in real time, several steps are expected. To have the approach applicable on real time systems in different stages, steps which have a better time complexity are utilized. This part nominally includes the following steps: 
i. Intensity of the plate modification

ii. Edge detection

iii. Separating objects from background

iv. Finding connected component

v. Candidate selection

\section{- Intensity modification}

Intensity modification help to enhance and somehow sharpening the license plate in order to have a better edge detection, histogram equalization is used to increase the intensity level range of the plate.

\section{- $\quad$ Edge detection}

In this step, the canny edge detection operator is utilized for edge detection as shown in Fig4. It is very important to take an account into the fact that the used of more robust algorithm for edge detection is very paramount like Canny's method, but the used of another edge operator like Sobel or Prewitt is not an offense since we do not need all of the edges within image.

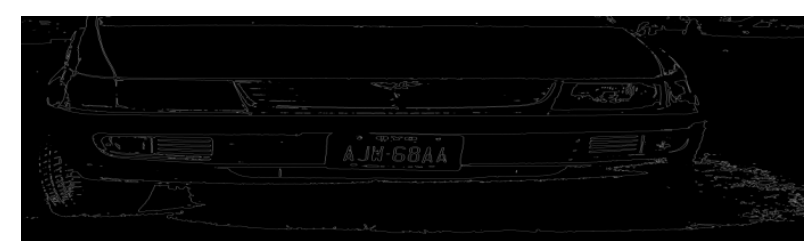

Figure 4: Canny edge detection

- $\quad$ Separating objects from background

In this step, thresholding is best used in separating objects from background as shown in Figure 5. Threshold is used to select rows with particularly white pixel density. Thresholding is a process used to separate object and background information. The method is based on converting a grayscale license plate image into a binary image. The conversion can be done in two types of threshold operation such as global thresholding and local thresholding. For the benefit of this research work otsu's thresholding was adopted which is a local thresholding.

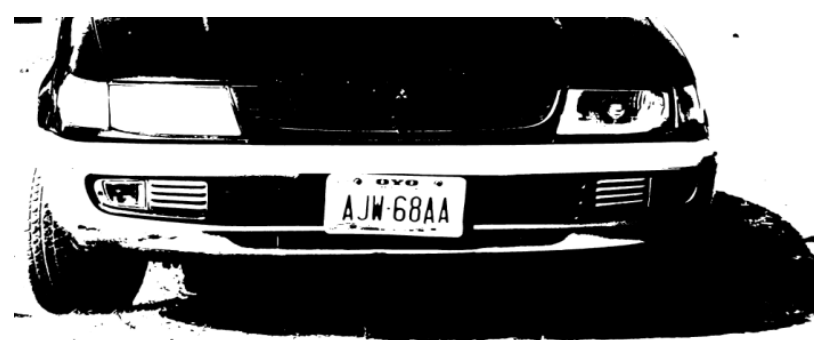

Figure 5: Thresholding

- $\quad$ Finding connected component

The next step is to find connected components. The connected objects were investigated using 8 and 4-ary connectivity. However, only 8-connected objects contain the desired regions of interest.

\section{- Candidate selection}

After successfully finding the connected components, the area of the plate, aspect ratio and the density of the region of the license plate were checked for other remaining objects in the image. The result achieved from these features is that components other than the probable number plate designate were deleted from the license plate area. Thereafter, the bounding box around object was calculated with the coordinates, which is the actual coordinates of the vehicle license plate.

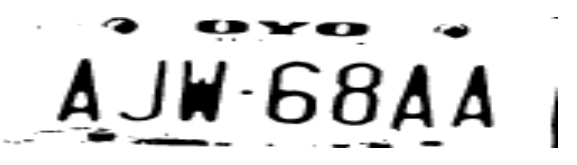

Figure 6: Actual vehicle license plate.

\subsection{Character segmentation}

Segmentation is one of the most important steps in the license plate recognition, character segmentation helped in achieving accurate character recognition. If the segmentation fails, it hinders recognition accuracy. Bounding box technique was used in segmenting detected vehicle license plate.

\subsection{Character Recognition}

This stage is a stage to recognize the detected and segmented vehicle license plate. Artificial Neural Network (ANN) was used to recognize the characters on the plate image.

\section{- $\quad$ Artificial Neural Network (ANN)}

ANNs have the ability to model linear and non-linear systems without the need to make assumptions implicitly as in most traditional statistical approaches. In this research work MATLAB neural network toolbox was used. Using neural network in OCR facilitates system extensibility i.e. ability to recognize more character sets than initially defined. In the character recognition algorithm using neural networks, the weights of the neural network were adjusted by training it using back propagation algorithm.

\section{Back Propagation (Bp) Algorithm}

Back propagation algorithm is a popularly used algorithm, and it can map non-linear processes. It is a feed forward network with one or more hidden layers. The elementary architecture of the back propagation network has three layers. There are no constraints about the number of hidden layers. Back propagation is a systematic method for training multilayer artificial neural networks. It has a mathematical expression that is very strong. The network must memorize all the training data (0-9) and (A-Z) which summed up to 36 characters. For the validation of the network, a software was developed that reads the sequence of characters, identify each character and recognize the identified character.

\section{EXPERIMENTALRESULTS}

Experiments were performed to test the recognition system and to measure the success rate. The method was implemented using MATLAB version 8.0 and configuration of personal computer includes: windows 8, Intel(R) Corei5, $2.3 \mathrm{GHz}$ processor with 4GB RAM. The images for the input to the system are captured coloured images. The test images were taken under varying Illumination conditions with three categories of plate numbers. The results are as given in tables 1,2 and 3 . 
International Journal of Advanced Research in Computer and Communication Engineering Vol. 4, Issue 11, November 2015

Table 1: Table showing results generated for license plate extraction at different categories.

(a) Category1 (The VLP is visible enough and its characters are clear and distinct).

\begin{tabular}{|c|c|c|c|c|c|c|}
\hline Threshold & Test No Plate & $\mathrm{TP}$ & FP & Precision & Success rate $(\%)$ & Computational time (sec) \\
\hline 0.20 & 40 & 38 & 2 & 0.95 & 95 & 3.509 \\
\hline 0.40 & 40 & 39 & 1 & 0.98 & 98 & 3.546 \\
\hline 0.60 & 40 & 39 & 1 & 0.98 & 98 & 3.525 \\
\hline 0.80 & 40 & 39 & 1 & 0.98 & 98 & 3.529 \\
\hline
\end{tabular}

(b) Category 2 (The vehicle images with little blur due to variation in illumination condition).

\begin{tabular}{|lccrrcc|}
\hline Threshold & Test No Plate & TP & FP & Precision & Success rate $(\%)$ & Computational time (sec) \\
\hline 0.20 & 20 & 18 & 2 & 0.90 & 90 & 1.586 \\
0.40 & 20 & 18 & 2 & 0.90 & 90 & 1.774 \\
0.60 & 20 & 19 & 1 & 0.95 & 95 & 1.530 \\
0.80 & 20 & 19 & 1 & 0.95 & 95 & 1.425 \\
\hline
\end{tabular}

(c) Category 3 (The vehicle images in these samples have little dirt or shadows on VLP region).

\begin{tabular}{|lcccccc|}
\hline Threshold & Test No Plate & TP & FP & Precision & Success rate (\%) & Computational time (sec) \\
\hline 0.20 & 10 & 7 & 3 & 0.70 & 70 & 0.503 \\
0.40 & 10 & 7 & 3 & 0.70 & 70 & 0.522 \\
0.60 & 10 & 7 & 3 & 0.70 & 70 & 0.520 \\
0.80 & 10 & 8 & 2 & 0.80 & 80 & 0.479 \\
\hline
\end{tabular}

Table 2: Table showing results generated for license plate recognition at different categories. (a) Category 1

\begin{tabular}{|c|c|c|c|c|c|c|}
\hline Threshold & Total character & rs $\quad$ TP & FP & Precision & Success rate $(\%)$ & Recognition time (sec) \\
\hline 0.20 & 320 & 289 & 31 & 0.9031 & 90.31 & 3.626 \\
\hline 0.40 & 320 & 299 & 21 & 0.9343 & 93.43 & 3.684 \\
\hline 0.60 & 320 & 305 & 15 & 0.9531 & 95.31 & 3.624 \\
\hline 0.80 & 320 & 308 & 12 & 0.9625 & 96.25 & 3.620 \\
\hline
\end{tabular}

(b) Category 2

\begin{tabular}{|c|c|c|c|c|c|c|}
\hline Threshold & Total cha & ers $\mathrm{T}$ & FP & Precision & Success rate $(\%)$ & Recognition time (sec) \\
\hline 0.20 & 160 & 122 & 38 & 0.7625 & 76.25 & 1.2503 \\
\hline 0.40 & 160 & 135 & 25 & 0.8437 & 84.37 & 1.1492 \\
\hline 0.60 & 160 & 141 & 19 & 0.8812 & 88.12 & 1.1417 \\
\hline 0.80 & 160 & 145 & 15 & 0.9062 & 91.00 & 1.1107 \\
\hline
\end{tabular}

(c) Category 3

\begin{tabular}{|c|c|c|c|c|c|c|}
\hline Threshold & Total cha & $\mathrm{rs} \quad \mathrm{TP}$ & FP & Precision & Success rate $(\%)$ & Recognition time (sec) \\
\hline 0.20 & 80 & 62 & 18 & 0.7750 & 77.50 & 0.4891 \\
\hline 0.40 & 80 & 68 & 12 & 0.8500 & 85.00 & 0.4808 \\
\hline 0.60 & 80 & 71 & 9 & 0.8875 & 88.75 & 0.4817 \\
\hline 0.80 & 80 & 73 & 7 & 0.9125 & 91.25 & 0.4900 \\
\hline
\end{tabular}

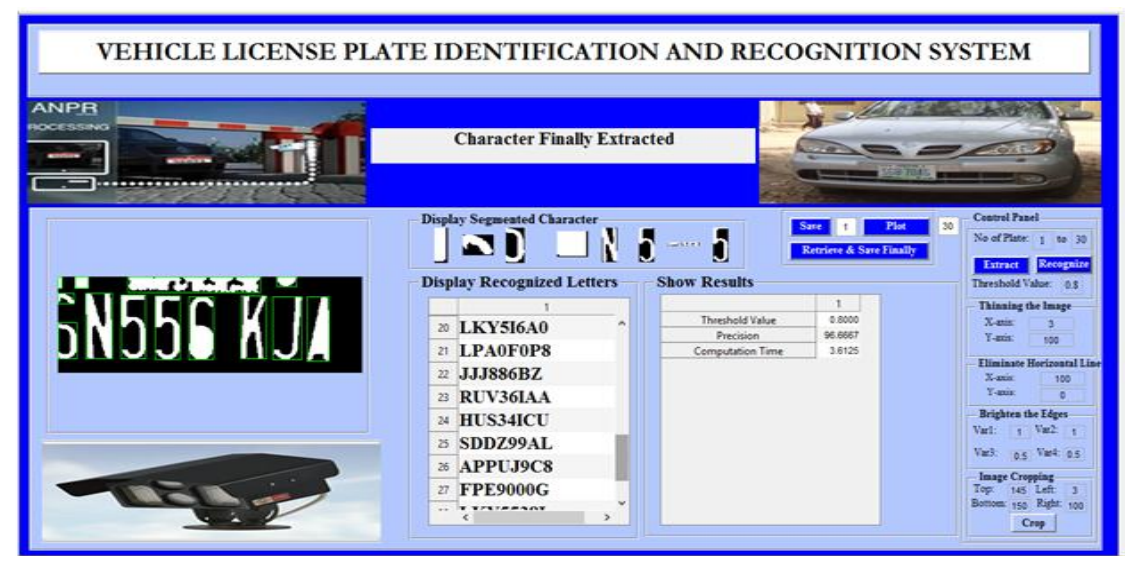

Figure 7: MATLAB GUI of the developed system 
Figure 7 depict the GUI of the developed system in 8 MATLAB.

The performance of the system was evaluated using the precision metric, where True Positive (TP) and False Positive are as defined.

$$
\text { Precision }=\frac{\mathrm{TP}}{\mathrm{TP}+\mathrm{FP}} \quad 1
$$

TP: Number of characters that are correctly detected or recognized.

FP: Number of characters that are wrongly detected or recognized.

\section{DISCUSSION OF RESULTS}

The performance of the developed recognition system were computed from the value of TP, FP obtained and summarized in Tables 1 and 2 . The precision was found to be $98 \%$ and $96 \%$ for number plate extraction and recognition respectively for category $1,95 \%$ and $91 \%$ for category 2, while category 3 has $80 \%$ in number plate extraction and $91 \%$ recognition success rate at the optimum threshold of 0.8 .

\section{CONCLUSION}

This paper presented an efficient technique for localization and recognition of Vehicle License Plates. The technique used was tested with 200 image samples and gives satisfactory performance. The applied technique was tested with still images in MATLAB tool box. For localization of VLP from the entire image, series of techniques was employed: plate intensity was enhanced using histogram equalization, edge detection was done with canny edge detection technique, separation of object from context using thresholding method, connected components were found and plate area selected and extracted while Artificial Neural network approach was used for character recognition.

\section{REFERENCES}

1. Plamondon R and Srihari S.N, "On-line and off- line handwritten character recognition: A comprehensive survey", IEEE. Transactions on Pattern Analysis and Machine Intelligence, vol. 22, no. 1, pp. 63-84, 2000.

2. Kahraman F, Kurt B, andGkmen M, "LicensePlate Character Segmentation Basedon the Gab or Transform and Vector Quantization,'Springler- Verlag Berlin Heidelberg, vol.2869, pp.381-388, 2003.

3. Mahbubul A .J, Khaled M, Tasnuva A, Mohsina K, and Bulbul A. "Bangla automatic number plate recognition system using artificial neural network" Asian Transactions on Science \& Technology (ATST ISSN: 2221-4283) Volume 02 Issue 01.2012

4. Arulogun O.T, Amusan D. G, "vehicle license plate recognition using edge detection and neural network" International Journal of Science and Research (IJSR) ISSN (Online): 2319-7064, 2015

5. Diegert C, "A combinatorial method for tracing objects using semantics of their shape," in Applied Imagery Pattern Recognition Workshop (AIPR), 2010 IEEE 39th, 2010, pp. 1-4. 2010

6. Sarfraz, M., Ahmed, M. J., Ghazi, S. A., "Saudi Arabian License Plate Recognition System", 2003 International Conference on Geometric Modeling and Graphics, 16-18 July 2003, pp.36-41, 2003.

7. Shanhong Zhu and Pei Tang "Color license plate localization algorithm research based on moiré principle analysis" 2013, Journal of Chemical and Pharmaceutical Research, 2014, 6(5): 706-709.
Rafael and Richard," "Digital Image Processing", 3rd ed. PrenticeHall Inc.2009

9. Cho D.U and Cho Y. H, "Implementation of pre-processing independent of environment and recognition of car number plate using histogram and template matching", The Journal of the Korean Institute of Communication Sciences, 23(1).

10. Ming-Kan Wu, et al., , "2-Level-Wavelet-Based License Plate Edge Detection", $5^{\text {th }}$ International Conference on Information Assurance and Security, ICIAS, pp.385-388.2009

\section{BIOGRAPHY}

Amusan D.G. is an E-Tutor at Open and Distance Learning Centre of Ladoke Akintola University of Technology, Ogbomoso, Oyo State, Nigeria. He graduated with B.Tech Computer Engineering from Ladoke Akintola University of Technology, Ogbomoso, Nigeria in the year 2010 and he is presently pursuing his masters in the same institution. His research interests are Intelligent Transportation Systems, Character \& Pattern Recognition, image processing, data and Information Security. 